\title{
The Liquid-Bridge with Large Gap in Micro Structural Systems
}

\author{
Shiqiao Gao, Lei Jin, Jingqing Du, Haipeng Liu \\ School of Mechatronic Engineering, Beijing Institute of Technology, Beijing, China \\ E-mail: gaoshq@bit.edu.cn \\ Received April 8, 2010; revised March 12, 2011; accepted March 17, 2011
}

\begin{abstract}
Based on the analysis of the total free energy of the liquid-bridge, several methods are presented to analyze the pull-off force of liquid-bridge. For the liquid bridge system with a large gap width, accurate solutions of a two-plate liquid bridge and a sphere-plane liquid bridge are given. In addition, the edge-effect resulting from the profile of the top solid in the liquid-bridge system is analyzed and calculated. It is proved by the subsequent tests.
\end{abstract}

Keywords: Liquid-Bridge, MEMS, Capillary, Pull-Off Force

\section{Introduction}

When a small amount of liquid is introduced at the small gap between two solid surfaces, a liquid-bridging phenomenon can be observed. Such a liquid-bridging phenomenon occurs in many cases. For example, a small water between the tip and the substrate surface of atomic force microscopy (AFM) will form a liquid-bridge [1,2], some water films or other liquids will cause a liquid-bridge between the tip and sample of the scanning force microscope (SFM) [3], a protective lubricant thinfilm used over the surface of the disk to separate the head slider from the disk can also cause meniscus rings around contacting asperities and liquid bridges between the head and the disk [4], an adsorbed water droplet due to humidity between the cantilever beam and the substrate of micro accelerator (or RF-MEMS) will result in a liquid-bridge [5], similarly many liquid-bridges between combs and substrate or between the neighbor combs of micro gyroscope will be formed when there is some water. With miniaturization of system and microminiaturization of the structures and objects, adhesion caused by capillary appears to be one major problem during the assembly and/or fabrication of micro-components and the operation of system.

The liquid-bridge will cause a capillary interaction between the solid surface and the liquid. To separate two solid surfaces in a liquid-bridge system, a force is needed to overcome the capillary attractive force caused by the liquid. This force is usually called as pull-off force or capillary force (or adhesion force). Such a capillary force is too insignificant for normal macro mechanical system to consider. But it will play a significant role in a micro scale system, especially in a nanoscale system. This is because surface forces become more and more important when size diminishes and the objects are scaled down. Capillary force is one of the adhesion forces. Capillary force can cause the thin polymer beams of the 3D microstructures to deflect and collapse during the evaporative drying of structures from liquid resin [6]. Adhesion can also prevent micro structures like RF-MEMS, micro sensor, micro gyroscope, or any high aspect ratio structures from normal functioning.

Of course, from another point of view, capillary force between two objects can be also used to manipulate small object [7]. In such a case, the capillary force applied onto the object should be controllable. Nevertheless, the major opposing force to picking up and releasing parts becomes adhesion. In assembly, it is as important to pick up as to release the object. In macro-assembly, this problem does not exist, because usual grippers can be closed (i.e. the part is locked between the digits of the gripper) or open (and the gripping force falls to zero). In micro-assembly, adhesion provides a very small value for the "gripping" force. If the weight of the object is smaller than adhesion, the object cannot be released. Conversely, if adhesion is smaller than the object weight, the object cannot be picked up.

The liquid of a liquid-bridge may exist originally (i.e. placed previously or remained after fabrication). It may 
be also subsequently formed by the phase transition from a condensable vapor near saturation to liquid. Therefore, for a micro structural system, especially for a nanostructural system, whether there is original liquid or not, it is possible for a liquid-bridge to be formed when ambient humidity is relatively large. To avoid the formation of a liquid-bridge for a micro system or a nanoscale system, an appropriate packing with sufficient drying vacuum is necessary.

In order to understand the liquid-bridging phenomenon, much effort has been made by earlier researchers [8-11]. In the aspect of theoretical studies, there are two ways to establish the models. One approach to determining force is the macroscopic Laplace-Kelvin Equation based on the meniscus theory. The other way is to make numerical computations based on the molecular theories, including molecular dynamics and Monte Carlo simulations, integral Equation and density functional theories. The profile of the liquid shows meniscus. According to the Laplace-Young Equation, a negative pressure difference between the inside and the outside of the meniscus will be formed. This negative pressure difference will be also applied on the wetted surface of solids in terms of the Pascal's law that no pressure gradient can exist for static liquids. This macroscopic approach usually assumes that the meniscus shape can be described by two principal radii, and its volume remains unchanged as the gap width changes. Even though this approach is simple, it has been validated for relative large scale structures. However, for nanoscale problems, this approach is not appropriate because of finite molecular size effects that give large fluctuations in meniscus size and shape. To study the capillary force in such a nanoscale case, some analyses based on the molecular theory are needed. For micro scale problem, this conventional simple approach can not be directly used either. Orr, Scriven and Rivas [12] presented a detailed literature survey on the earlier work related to the meniscus properties and capillary force involved in the liquid bridging between two solid surfaces by means of solving the Laplace-Young Equation. They discussed the aspects of liquid mechanics, including the volume of liquid, surface area, and mean curvature of the meniscus, and the liquid-bridging forces exerted on the solid surfaces. Recently, on the one hand, instead of a rigid solid, some elastic deformations and the coupling interaction between solid and liquid have been considered and discussed by the well-known Hertzian solution for the contact between a sphere and a plane $[4,13]$. On the other hand, the effect of humidity on the capillary has been studied by many researchers [14]. In the same time, of course, some computational simulations have been made and developed based on the modern molecular theory [2,15], e.g. a density functional theory (DFT), the molecular dynamics (MD), the Monte Carlo (MC) method, the grand canonical MC (GCMC) method, and so on. In addition to the equilibrium method mentioned above, the energy method has also been used to solve the pull-off force [16]. Although the equilibrium method based on the Laplace-Young Equation is equivalent to the energy method, there are also some differences in detail between them because many factors have been neglected in the equilibrium method.

In the aspect of experiments, many studies have been also made by earlier researchers [8,17-20]. The earliest measurement was made by McFarlane and Tabor [8]. They measured the meniscus force of water in atmospheres with different relative humidities by measuring the pull-off force between a glass ball and a glass plane surface in contact. According to the conventional simple theory, the pull-off force is independent of the relative humidity. However, McFarlane and Tabor found in their experiments that the pull-off force decreased suddenly when the relative humidity was less than $90 \%$ and the decrease was dependent on the roughness of the glass surfaces. They concluded that the decrease would occur when the height of the surface roughness was comparable with the thickness of the adsorbed liquid film. Later, Fisher and Israelachvili [17] made a more precise measurement of the meniscus force for water, benzene, cyclohexane, $n$-hexane and 2-methylbutane in atmospheres of their own vapors at a relative vapor pressure in the range of $0 \%-99 \%$. They used molecularly smooth mica in contact surfaces instead of glass to avoid the effect of surface roughness on measurement. They found that for organic liquids the surface tension theory based on bulk thermodynamics was applicable even when the adsorbed film was only a few molecules in thickness. However, for water it was quite different. Their experimental results for water showed that the meniscus force due to the Laplace pressure reduced to $90 \%$ of that expected from bulk thermodynamics when the relative vapor pressures is 0.9 , corresponding to a Kelvin meniscus radius of about $5 \mathrm{~nm}$. They explained the results in terms of the assumption that the long-range cooperative nature of the hydrogen bonding interaction and electric double layer forces in water film between solid surfaces may play a role in reducing the effective surface tension of water. Christenson [18] modified the surface force apparatus by adopting a double cantilever spring. They obtained very different experimental results from those of Fisher and Israelachvili. They found that for organics such as cyclohexane and $n$-hexane the measured pull-off force increased with the decrease in the relative vapor pressure, and for water, although the measured pull-off force decreased as the relative vapor pressure decreased, the decrease was much smaller than that obtained by 
Fisher and Israelachvili. With the inventions of the scanning force microscope (SFM), scanning tunnel microscope (STM) [21], the atomic force microscope (AFM), scanning probe microscope (SPM), and the surface force apparatus (SFA), many powerful tools have been made and developed for observing surface morphology on an nano-scale and atomic scale. They make a powerful probe for a variety of surface studies available. They enable us to investigate various sample properties such as adhesion, surface charge, and magnetic properties on a nanometer scale. But on the other hand, it is unavoidable for them to face the liquid-bridge problems on nanometer scale.

From the earlier research worksmentioned above, whether the theoretical studies or the experimental studies, most of the investigated objects are the liquid-bridge system composed of a spherical tip and a plane substrate. Most of those studies were made with the constraint of small separating distance. When the wetted area is much larger than the gap between the two solids (i.e. large deep-wide ratio), the shape of meniscus can be negligible during calculating the volume of liquid. Even in the modified model established by Mingyan He, et al. [1], instead of the meniscus curve surface, a cylindrical surface was still used for calculation of the liquid volume. Nevertheless, when the gap becomes large, the shape of profile has an apparent effect on the volume and other geometrical parameters. From the theoretical analysis, it can be also apparently seen that the capillary force depends tightly on the geometric (shape) parameters of meniscus profile, such as the principle radii, the contact angles, the fulfilling angles, the volume, the interfacial areas, the gap width, and so on. In order to model the meniscus profile accurately, O. H. Pakarinen et al. [14] presented a method to numerically calculate the exact (non-circular) meniscus profile from the Kelvin Equation. But it is not analytical model. The computation based on the difference Equations was also approximate. For a micro scale problem, the conventional continuum model is too simple to predict the capillary force. But the computational simulation is too complicated to analyze. In view of this micro scale, this current article attempts to give a relatively accurate solution of the pull-off force of micro liquid-bridge by means of an analytical method based on the energy theory.

\section{The Total Free Energy of the Liquid-Bridge}

The surface tension is always trying to make the area contract. Therefore, with the increase of area, the potential energy will increase. On the contrary, the pressure is always trying to make the volume expand. Therefore, with the decrease of volume, the potential energy will increase. The total free energy consists of two parts which are free surface energy and free bulk energy respectively. The free surface energy arises from the contributions of the solid/liquid and liquid/vapor interfaces. The free bulk energy arises from the contributions of liquid and vapor volumes with corresponding pressures. In order to calculate the free energy of the liquid-bridging system, the liquid volume and the surface areas of the interfaces are need to be calculated. For a liquid bridge, the total free energy can be written by

$$
E=\sum_{i=1}^{n} \sigma_{i} A_{i}-\sum_{j=1}^{m} p_{j} V_{j}
$$

where the first sum term in right-hand side is the total free surface energy, the second sum term is the total free bulk energy, $A$ stands for interfacial area and $\sigma$ the interfacial tension, $V$ stands for the phase volume and $p$ the phase pressure, where the subscript $i$ stands for the interface and the subscript $j$ stands for the phase. Naturally there are usually three phases, which are vapor phase, liquid phase and solid phase respectively. The interface means the contact surface between every two phases, e.g. the interface between vapor phase and liquid phase, the interface between vapor phase and solid phase, and the interface between liquid phase and the solid phase. Liquid bridge is a system constructed by two solid phase, one liquid phase and one vapor phase. Therefore there are two vapor-solid interfaces, two liquid-solid interfaces and one vapor-liquid interface shown in Figure 1.

If the interface area between top solid and liquid is $A_{\text {Ist }}$ (top wetted area), the interface area between top solid and vapor is $A_{v s t}$, the interface area between substrate solid (bottom solid) and liquid is $A_{s b b}$ (bottom wetted area), the interface area between substrate solid and vapor is $A_{v s b}$, whereas the interface area between vapor and liquid (i.e. the profile of the liquid) is $A_{v v p}$, the volume of liquid is $V_{l}$, and the volume of vapor is

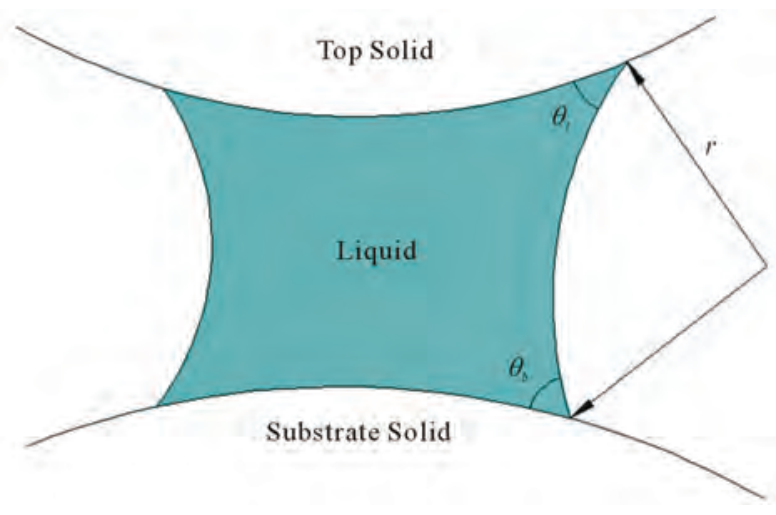

Figure 1. A general liquid-bridge. 
$V_{v}$, the total free energy of the liquid bridge system described by Equation (1) can be rewritten as

$$
\begin{aligned}
E= & \sigma_{l s t} A_{l s t}+\sigma_{v s t} A_{v s t}+\sigma_{l s b} A_{l s b}+\sigma_{v s b} A_{v s b} \\
& +\sigma_{l v p} A_{l v p}-p_{l} V_{l}-p_{v} V_{v}
\end{aligned}
$$

Because the interfacial tension between solid and vapor is very small, the effect of solid-vapor interface in Equation (2) can be neglected. Then, we have

$$
E=\sigma_{l s t} A_{l s t}+\sigma_{l s b} A_{l s b}+\sigma_{l v p} A_{l v p}-p_{l} V_{l}-p_{v} V_{v}
$$

When we solve some forces by means of the energy theory, it is needed to differentiate the total free energy. At a constant temperature, the differentiation of total free energy can be derived by

$$
\begin{aligned}
\delta E & =\sigma_{l s t} \delta A_{l s t}+\sigma_{l s b} \delta A_{l s b}+\sigma_{l v p} \delta A_{l v p} \\
& -p_{l} \delta V_{l}-p_{v} \delta V_{v v}
\end{aligned}
$$

Because the total volume of liquid phase and vapor phase is constant, i.e. $\delta\left(V_{l}+V_{v}\right)=0$, there is

$$
\delta V_{v}=-\delta V_{l}
$$

Substituting Equation (5) into Equation (4), leads to

$$
\delta E=\sigma_{l s t} \delta A_{l s t}+\sigma_{l s b} \delta A_{l s b}+\sigma_{l v p} \delta A_{l v p}-\left(p_{l}-p_{v}\right) \delta V_{l}
$$

For rigid solids, there is no deformation on the interfaces of solid and liquid. The interfaces have the same shapes as the solid surface. Nevertheless, the interface of liquid and vapor will keep meniscus shape with some corresponding curvatures due to the capillary effect.

\section{The Solution on the Interface of Liquid and Vapor of the Liquid-Bridge}

On the interface of liquid and vapor of the liquid bridge, i.e. the meniscus profile, excepting the pressure of vapor and the pressure of liquid, there is no other external force. In terms of work-energy conservation theory, there is

$$
\begin{aligned}
f_{l v p}=\frac{\delta E}{\delta r}= & \sigma_{l s t} \frac{\delta A_{l s t}}{\delta r}+\sigma_{l s b} \frac{\delta A_{l s b}}{\delta r}+\sigma_{l v p} \frac{\delta A_{l v p}}{\delta r} \\
& -\left(p_{l}-p_{v}\right) \frac{\delta V_{l}}{\delta r}=0
\end{aligned}
$$

where $r$ is the normal coordinate of the curve surface of the meniscus profile. Considering $\delta A_{l s t}=\delta A_{1 s b}=0$, we have

$$
\sigma_{l v p} \frac{\delta A_{l v p}}{\delta r}-\left(p_{l}-p_{v}\right) \frac{\delta V_{l}}{\delta r}=0
$$

Supposing the two principal radii of the curve surface of the meniscus profile are $r_{1}$ and $r_{2}$ respectively, by means of the geometric relationships of the spatial curve surface, we can obtain the following relationship

$$
\delta A_{l v p}=-\left(\frac{1}{r_{1}}+\frac{1}{r_{2}}\right) \delta V_{l}
$$

Substituting Equation (9) into Equation (8), leads to

$$
\left[\sigma_{l v p}\left(\frac{1}{r_{1}}+\frac{1}{r_{2}}\right)+\left(p_{l}-p_{v}\right)\right] \frac{\delta V_{l}}{\delta r}=0
$$

That is

$$
\Delta p=p_{v}-p_{l}=\sigma_{l v p}\left(\frac{1}{r_{1}}+\frac{1}{r_{2}}\right)
$$

This equation is the so called Young-Laplace Equation.

\section{The Pull-Off Force of the Top Solid in the Liquid-Bridge System}

\subsection{The Solution for Constant Wetted Areas}

To obtain the pull-off force of the top solid in the liquid bridge, a classical derivative of the energy with respect to the distance between the top and substrate solid objects (i.e. vertical coordinate $z$ ) can be used. This principle of work-energy conservation can be explained as follows. An increment displacement $\delta z$ in $z$ direction will result in an increment of total free energy of the liquid bridge system $\delta E$. In terms of the principle of work-energy conservation, this increment $\delta E$ of energy should arise from the work done by pull-off force $f_{a}$ in the distance of $\delta z$, that is $\delta E=f_{a} \cdot \delta z$, which leads to

$$
f_{a}=\frac{\delta E}{\delta z}
$$

Substituting Equation (6) into Equation (12), leads to

$$
\begin{aligned}
f_{a}= & \frac{\delta E}{\delta z}=\sigma_{l s t} \frac{\delta A_{l s t}}{\delta z}+\sigma_{l s b} \frac{\delta A_{l s b}}{\delta z}+\sigma_{l v p} \frac{\delta A_{l v p}}{\delta z} \\
& -\left(p_{l}-p_{v}\right) \frac{\delta V_{l}}{\delta z}
\end{aligned}
$$

In the case of constant interfacial areas, there are $\delta A_{l s t}=\delta A_{1 s b}=0$. To take liquid with a small chip from a cup of liquid belongs to this case shown in Figure 2. The increment of the profile area is

$$
\delta A_{l v p}=l_{t} \cdot \delta z \cdot \sin \theta_{t}
$$

where $\theta_{t}$ is the contact angle of the liquid with respect to the top solid, and $l_{t}$ is the perimeter of the interfacial (wetted) area $A_{l s t}$. Whereas $\delta V_{l}=A_{l s t} \cdot \delta \mathrm{z}$. Substituting them into Equation (13), leads to

$$
\begin{aligned}
f_{a} & =\frac{\delta E}{\delta z}=\sigma_{l v p} \cdot l_{t} \cdot \sin \theta_{t}-\left(p_{l}-p_{v}\right) \cdot A_{l s t} \\
& =\sigma_{l v p} \cdot l_{t} \cdot \sin \theta_{t}+\Delta p \cdot A_{l s t}
\end{aligned}
$$




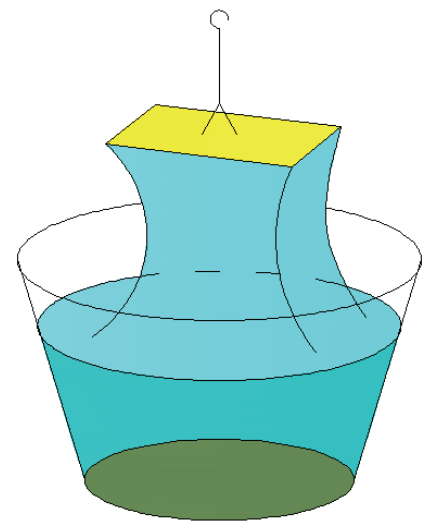

Figure 2. Taking liquid with a piece of thin plate from a cup.

Taking $\sigma_{l v p}=\sigma$ (the surface tension) and $\Delta p=\frac{2 \sigma}{r_{m}}$ where $r_{m}$ is the mean radii of the meniscus profile where $2 / r_{m}=1 / r_{1}+1 / r_{2}$, leads to

$$
f_{a}=\sigma \cdot l_{t} \cdot \sin \theta_{t}+\frac{2 \sigma}{r_{m}} \cdot A_{l s t}
$$

\subsection{An Approximate Solution for Constant Liquid Volume}

For a general liquid bridge, the liquid keeps a constant volume, that is $\delta V_{l}=0$. Equation (13) can be rewritten by

$$
f_{a}=\frac{\delta E}{\delta z}=\sigma_{l s t} \frac{\delta A_{l s t}}{\delta z}+\sigma_{l s b} \frac{\delta A_{l s b}}{\delta z}+\sigma_{l v p} \frac{\delta A_{l v p}}{\delta z}
$$

To obtain the solution of Equation (17), some geometric relationships need to be analyzed and discussed. We define a deep-wide ratio as $\zeta=d / w$ where $d$ is thickness of liquid film or the minimum gap width between the two solid objects and $w$ is the length in maximum wide direction of area $A_{l s t}$. If the liquid bridge has a small deep-wide ratio, i.e. $\zeta \ll 1$ and $A_{1 s t} \approx A_{1 s b}$, the volume of liquid can be approximately expressed as

$$
V_{l} \approx d \cdot A_{l s t} \approx d \cdot A_{l s b}
$$

Considering the volume as a constant (isovolume), we have

$$
\delta V_{l} \approx d \cdot \delta A_{l s t}+\delta d \cdot A_{l s t}=d \cdot \delta A_{l s t}+\delta z \cdot A_{l s t}=0
$$

Therefore, there is

$$
\frac{\delta A_{t s t}}{\delta z}=-\frac{A_{t s t}}{d}
$$

Similarly there is

$$
\frac{\delta A_{1 s b}}{\delta z}=-\frac{A_{l s b}}{d}
$$

From the geometric relationship of meniscus profile of the liquid bridge shown in Figure 3, the relationship between the thickness of liquid film $d$ (the distance between the two solid objects) and the curvature radius $r_{1}$ can be written by

$$
d=r_{1}\left(\cos \theta_{t}+\cos \theta_{b}\right)
$$

where $\theta_{b}$ is the contact angle of the liquid with respect to the substrate solid.

Substituting Equations (14), (20) and (21) into Equation (17) and noting that $\sigma_{l s t}=-\sigma \cos \theta_{t}, \sigma_{l s b}=-\sigma \cos \theta_{b}$ and $\sigma_{l v p}=\sigma$, leads to

$$
f_{a}=\frac{\delta E}{\delta z}=\sigma\left(\cos \theta_{t}+\cos \theta_{b}\right) \frac{A_{l s t}}{d}+\sigma \sin \theta_{t} l_{t}
$$

Substituting Equation (22) into Equation (23), leads to

$$
f_{a}=\sigma \frac{A_{1 s t}}{r_{1}}+\sigma \sin \theta_{t} l_{t}
$$

\subsection{An Accurate Solution for Large Gap Width}

If the deep-wide ratio is not small, a more general model should be built. If the top and substrate solids are all planes shown in Figure 4, the increment of the vertical distance can be also written as

$$
\delta z=\delta r_{1}\left(\cos \theta_{t}+\cos \theta_{b}\right)
$$

Whereas the increment of volume should be written as

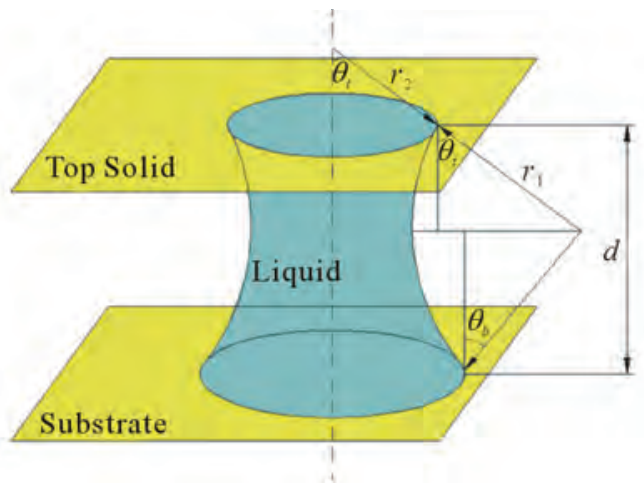

Figure 3. A circular liquid bridge.

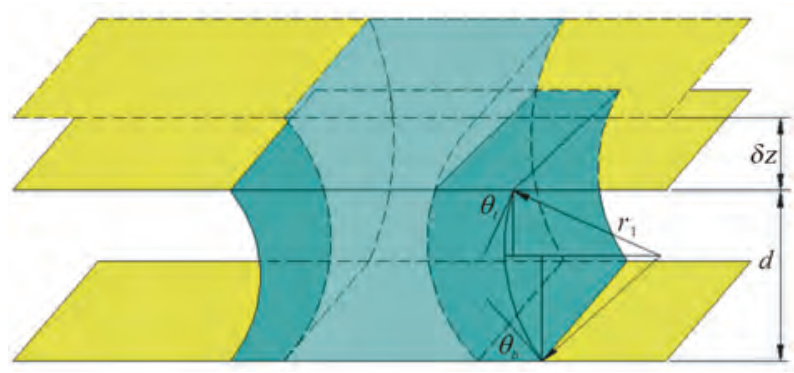

Figure 4. A rectangular liquid bridge. 


$$
\delta V_{l}=-A_{l v p} \delta x+A_{l s t} \delta z
$$

Because the volume keeps a constant, there is $\delta V_{l}=0$. Therefore we have

$$
\delta x=\frac{A_{l s t}}{A_{l v p}} \delta z
$$

In this case, the area change of top interface can be written as

$$
\delta A_{\text {Ist }}=-l_{t} \cdot \delta x=-\frac{l_{t} A_{i s t}}{A_{\text {ivp }}} \delta z
$$

The area change of substrate interface can be written as

$$
\delta A_{t s b}=-l_{b} \cdot \delta x=-\frac{l_{b} A_{s b b}}{A_{t w p}} \delta z
$$

where $l_{t}$ and $l_{b}$ are perimeters of the top interface and the substrate interface respectively.

The change of profile area can be written as

$$
\delta A_{l v p}=l_{t} \cdot \sin \theta_{t} \cdot \delta z
$$

Substituting Equation (28), (29) and (30) into Equation (17) and noting $\sigma_{l s t}=-\sigma \cos \theta_{t}, \sigma_{l s b}=-\sigma \cos \theta_{b}$ and $\sigma_{l v p}=\sigma$, leads to

$$
f_{a}=\frac{\delta E}{\delta z}=\sigma\left(l_{t} \cos \theta_{t}+l_{b} \cos \theta_{b}\right) \frac{A_{t s t}}{A_{l v p}}+\sigma \sin \theta_{t} l_{t}
$$

For a polygonal interface as shown in Figure 5, the area of profile for one side can be written by means of integral as

$$
\begin{aligned}
A_{i}= & \int_{-\left(\frac{\pi}{2}-\theta_{b}\right)}^{\frac{\pi}{2}-\theta_{t}} 2 \tan \frac{\alpha_{i}}{2}\left[\frac{l_{t i}}{2} \tan ^{-1} \frac{\alpha_{i}}{2}\right. \\
& \left.+r_{1} \cos \left(\frac{\pi}{2}-\theta_{t}\right)-r_{1} \cos \beta\right] r_{1} \mathrm{~d} \beta
\end{aligned}
$$

where $\alpha_{i}$ is the central angle of $i^{\text {th }}$ th side and $l_{t i}$ is the length of the $i^{\text {th }}$ side. Integrating Equation (32) and summing all the sides, leads to

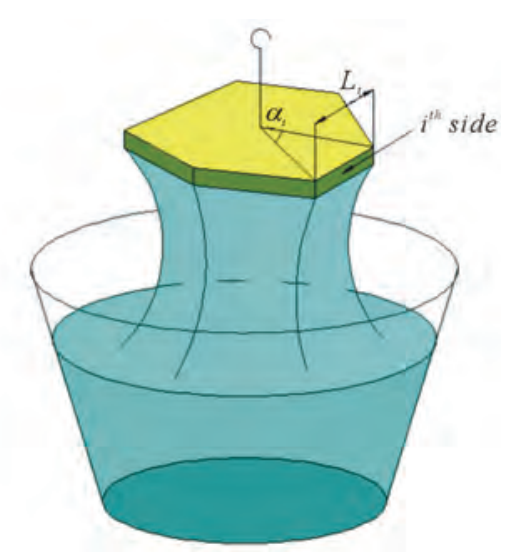

Figure 5. A polygonal liquid bridge.

$$
\begin{aligned}
A_{\text {lvp }}= & \sum_{i=1}^{n} A_{i} \\
= & \sum_{i=1}^{n} 2 \tan \frac{\alpha_{i}}{2}\left[\left(\frac{l_{t i}}{2} \tan ^{-1} \frac{\alpha_{i}}{2}\right.\right. \\
& \left.\left.+r_{1} \sin \theta_{t}\right) r_{1}\left(\pi-\theta_{t}-\theta_{b}\right)-r_{1}^{2}\left(\cos \theta_{t}+\cos \theta_{b}\right)\right]
\end{aligned}
$$

For a circular interface as shown in Figure 3, taking the limit of $\alpha_{i} \rightarrow 0$ and $n \rightarrow \infty$, leads to

$$
\begin{aligned}
A_{\text {vvp }} & =\lim _{\alpha_{i} \rightarrow 0(n \rightarrow \infty)} \sum_{i=1}^{n} A_{i} \\
& =\int_{0}^{2 \pi}\left[\left(r_{2} \sin \theta_{t}+r_{1} \sin \theta_{t}\right) r_{1}\left(\pi-\theta_{t}-\theta_{b}\right)\right. \\
& \left.-r_{1}^{2}\left(\cos \theta_{t}+\cos \theta_{b}\right)\right] \mathrm{d} \alpha \\
& =2 \pi r_{1}\left[\left(r_{2}+r_{1}\right) \sin \theta_{t}\left(\pi-\theta_{t}-\theta_{b}\right)\right. \\
& \left.-r_{1}\left(\cos \theta_{t}+\cos \theta_{b}\right)\right]
\end{aligned}
$$

For a rectangular interface shown in Figure 6, the profile area is derived by

$$
\begin{aligned}
A_{\text {vvp }}= & \sum_{i=1}^{n} A_{i}=4\left\{\frac { b } { a } \left[\left(\frac{a}{2}+r_{1} \sin \theta_{t}\right) r_{1}\left(\pi-\theta_{t}-\theta_{b}\right)\right.\right. \\
& \left.-r_{1}^{2}\left(\cos \theta_{t}+\cos \theta_{b}\right)\right] \\
& +\frac{a}{b}\left[\left(\frac{b}{2}+r_{1} \sin \theta_{t}\right) r_{1}\left(\pi-\theta_{t}-\theta_{b}\right)\right. \\
& \left.\left.-r_{1}^{2}\left(\cos \theta_{t}+\cos \theta_{b}\right)\right]\right\}
\end{aligned}
$$

where $a$ and $b$ are respectively length and width of the rectangle.

For a square interface, there is

$$
\begin{aligned}
A_{\text {lvp }} & =\sum_{i=1}^{n} A_{i}=\left[\left(l_{t}+8 r_{1} \sin \theta_{t}\right) r_{1}\left(\pi-\theta_{t}-\theta_{b}\right)\right. \\
& \left.-8 r_{1}^{2}\left(\cos \theta_{t}+\cos \theta_{b}\right)\right]
\end{aligned}
$$

It should be pointed out that, for a narrow-long rectangular solid shown in Figure 6, the interface area will

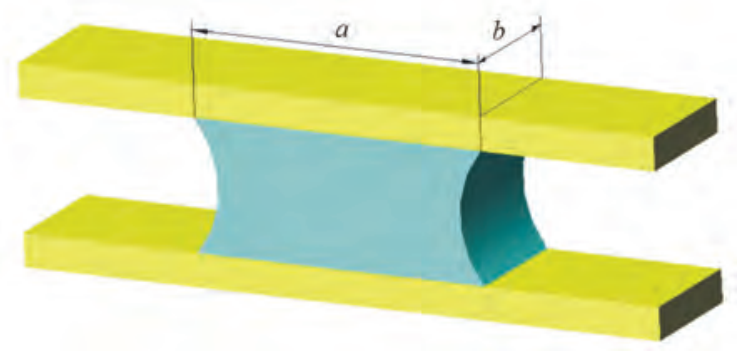

Figure 6. A narrow-long rectangular liquid bridge. 
firstly contract apart from the long side until to form a square shape, and then will contract apart from the short side until to form a circler shape.

Substituting Equation (34) into Equation (31), leads to* Equation (37)

If the material of top solid is the same as that of substrate solid, there are $\theta_{t}=\theta_{b}$ and $l_{t}=l_{b}$. Then there is

$$
\begin{aligned}
f_{a}= & \sigma \frac{2 l_{t} \cos \theta_{t} A_{s t}}{r_{1}\left(l_{t}+2 \pi r_{1} \sin \theta_{t}\right)\left(\pi-2 \theta_{t}\right)-4 \pi r_{1} \cos \theta_{t}} \\
& +\sigma \sin \theta_{t} l_{t}
\end{aligned}
$$

For a liquid bridge with small deep-wide ratio, $l_{t} . \gg$ $2 r_{1} \cos \theta_{t}$. Equation (38) can be rewritten as

$$
f_{a}=\sigma \frac{2 \cos \theta_{t} A_{l s t}}{r_{1}\left(\pi-2 \theta_{t}\right)}+\sigma \sin \theta_{t} l_{t}
$$

Comparing Equation (24) with Equation (39), it is found that these two Equations are not the same. It should be said that Equation (39) is a more accurate Equation in which the curve surface of profile has been considered during the calculation of liquid volume. If we use the straight line distance $2 r_{1} \cos \theta_{t}$ instead of the curve arc distance $r_{1}\left(\pi-2 \theta_{t}\right)$, Equation (39) will change to Equation (24). For a square interface, substituting Equation (36) into Equation (31) and considering $l_{t}$. $2 r_{1} \cos \theta_{t}$, the same Equation as (39) can be obtained.

It must be pointed out that, with the contracting of solid-liquid interface apart from every boundary side, the shape of interface tends to a circle acted by the surface tension force.

\subsection{An Accurate Solution for the Sphere-Plane Liquid Bridge System with a Large Gap Width}

When the top solid has the spherical shape and the substrate is plane shown in Figure 7(a), the problem becomes relatively complicated. The conservation condition of volume can be also written by

$$
\delta V_{l}=-A_{l v p} \delta x+A_{l s t} \delta z=0
$$

Then there is

$$
\delta x=\frac{A_{l s t}}{A_{l v p}} \delta z
$$

When the sphere moves upward with a tiny displacement $\delta z$, the meniscus interface of liquid-vapor will also change, including the curvature center and the cur- vature radius. But the contact angles should keep constant. Supposing the curvature center has a tiny displacement in left-hand direction $\delta x_{0}$ and a tiny upward displacement $\delta y_{0}$, and the curvature radius has an increment $\delta r$, the wetted boundary on the spherical interface will contract with a displacement $\delta x_{t}$ in left-hand direction, which can be written as

$$
\delta x_{t}=\delta x_{0}+\delta r \sin \left(\theta_{t}+\varphi\right)-r \cos \left(\theta_{t}+\varphi\right) \delta \varphi
$$

where $\varphi$ is called the filling angle and $\delta \varphi$ is defined as positive in clockwise direction.

The vertical upward displacement can be written as

$$
\delta y_{t}=\delta y_{0}+\delta r \cos \left(\theta_{t}+\varphi\right)+r \sin \left(\theta_{t}+\varphi\right) \delta \varphi
$$

From the geometric relationships shown in Figure 7, the displacement of meniscus curve surface of profile (or the displacement in left-hand direction of the horizontal apex of the profile) can be written as

$$
\delta x=\delta x_{0}+\delta r
$$

The wetted area on the substrate plane will contract with a following decrement

$$
\delta \rho=\delta x-\left(1-\sin \theta_{b}\right) \delta r
$$

Because there are $\delta x_{t}=R \cos \varphi \delta \varphi$ and $\delta y_{0}=\delta r \cos \theta_{b}$, we can obtain the following relationships

$$
\delta z=\delta y_{t}+R \sin \varphi \delta \varphi
$$

and

$$
\delta y_{t}=\left[\cos \theta_{b}+\cos \left(\theta_{t}+\varphi\right)\right] \delta r+r \sin \left(\theta_{t}+\varphi\right) \delta \varphi
$$

Substituting Equation (47) into Equation (46), leads to

$$
\begin{aligned}
\delta z & =\left[\cos \theta_{b}+\cos \left(\theta_{t}+\varphi\right)\right] \delta r \\
& +\left[R \sin \varphi+r \sin \left(\theta_{t}+\varphi\right)\right] \delta \varphi
\end{aligned}
$$

Substituting Equation (42) into Equation (44), leads to

$$
\begin{aligned}
\delta x & =\left[1-\sin \left(\theta_{t}+\varphi\right)\right] \delta r \\
& +\left[R \cos \varphi+r \cos \left(\theta_{t}+\varphi\right)\right] \delta \varphi
\end{aligned}
$$

From the Equations (41), (45), (47) and (48), the following solutions can be obtained

$$
\frac{\delta r}{\delta z}=\frac{C_{1}-C_{3} \xi}{C_{1} C_{2}-C_{3} C_{4}}
$$

and

$$
\frac{\delta \varphi}{\delta z}=\frac{C_{2} \xi-C_{4}}{C_{1} C_{2}-C_{3} C_{4}}
$$

$$
\text { * } f_{a}=\sigma \frac{\left(l_{t} \cos \theta_{t}+l_{b} \cos \theta_{b}\right) A_{l s t}}{r_{1}\left(l_{t}+2 \pi r_{1} \sin \theta_{t}\right)\left(\pi-\theta_{t}-\theta_{b}\right)-2 \pi r_{1}\left(\cos \theta_{t}+\cos \theta_{b}\right)}+\sigma \sin \theta_{t} l_{t}
$$




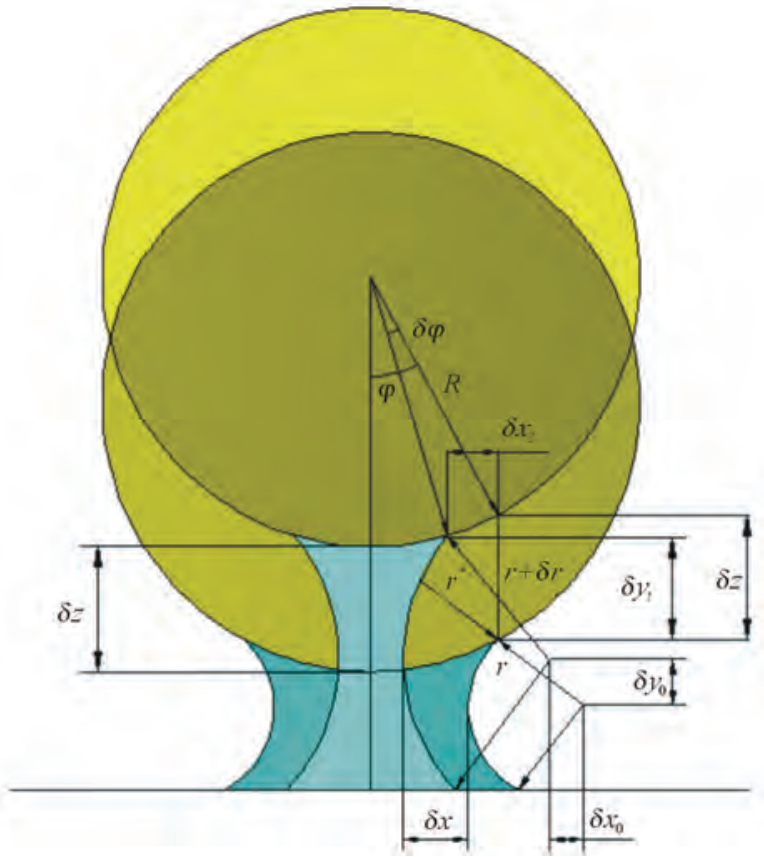

(a)

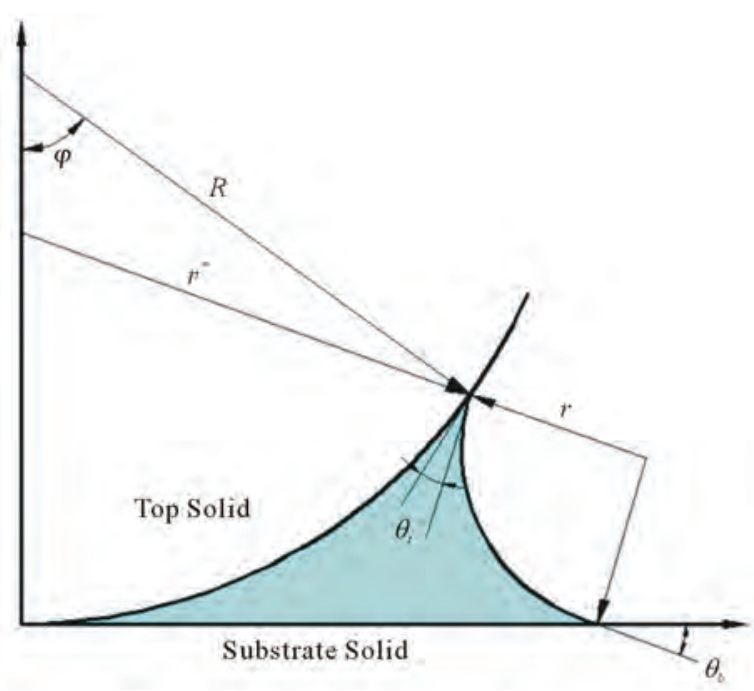

(b)

Figure 7. A sphere-plane liquid bridge system with a large gap width.

and

where

$$
\frac{\delta \rho}{\delta z}=\xi-\frac{C_{5}\left(C_{1}-C_{3} \xi\right)}{C_{1} C_{2}-C_{3} C_{4}}
$$

$$
\begin{aligned}
& C_{1}=R \cos \varphi+r \cos \left(\theta_{t}+\varphi\right), C_{2}=\cos \theta_{b}+\cos \left(\theta_{t}+\varphi\right), \\
& C_{3}=R \sin \varphi+r \sin \left(\theta_{t}+\varphi\right), C_{4}=1-\sin \left(\theta_{t}+\varphi\right), \\
& C_{5}=1-\sin \theta_{b} \text { and } \xi=A_{l s t} / A_{l v p} .
\end{aligned}
$$

The wetted area on the sphere can be written as

$$
A_{l s t}=2 \pi R^{2}(1-\cos \varphi)
$$

The wetted area on the substrate plane can be written as

$$
A_{l s b}=\pi \rho^{2}
$$

The increment of meniscus area of profile can be described as

$$
\delta A_{\text {lvp }}=2 \pi R \sin \varphi \delta s
$$

where $\delta$ s is the increment of the meridian of the profile, which can be derived as

$$
\delta s=r \delta \varphi+\delta r\left(\pi-\theta_{t}-\theta_{b}-\varphi\right)
$$

By means of Equations (50), (51) and (52), deriving Equations (53), (54) and (55) with respect to the vertical coordinate $z$, leads to

$$
\begin{aligned}
\frac{\delta A_{l s t}}{\delta z} & =-\frac{2 \pi R^{2} \sin \varphi\left(C_{2} \xi-C_{4}\right)}{C_{1} C_{2}-C_{3} C_{4}} \\
\frac{\delta A_{l s b}}{\delta z} & =-2 \pi \rho\left[\xi-\frac{C_{5}\left(C_{1}-C_{3} \xi\right)}{C_{1} C_{2}-C_{3} C_{4}}\right]
\end{aligned}
$$

$$
\begin{aligned}
& \frac{\delta A_{\text {lvp }}}{\delta z}= \\
& 2 \pi R \sin \varphi \frac{r\left(C_{2} \xi-C_{4}\right)+\left(C_{1}-C_{3} \xi\right)\left(\pi-\theta_{t}-\theta_{b}-\varphi\right)}{C_{1} C_{2}-C_{3} C_{4}}
\end{aligned}
$$

Substituting Equations (57), (58) and (59) into Equation (17) and noting $\sigma_{l s t}=-\sigma \cos \theta_{t}, \quad \sigma_{l s b}=-\sigma \cos \theta_{b}$ and $\sigma_{l v p}=\sigma$, leads to

$$
\begin{aligned}
& f_{a}=\frac{2 \pi R^{2} \sin \varphi\left(C_{2} \xi-C_{4}\right)}{C_{1} C_{2}-C_{3} C_{4}} \sigma \cos \theta_{t} \\
& +2 \pi \rho\left[\xi-\frac{C_{5}\left(C_{1}-C_{3} \xi\right)}{C_{1} C_{2}-C_{3} C_{4}}\right] \sigma \cos \theta_{b} \\
& +2 \pi R \sin \varphi \frac{r\left(C_{2} \xi-C_{4}\right)+\left(C_{1}-C_{3} \xi\right)\left(\pi-\theta_{t}-\theta_{b}-\varphi\right)}{C_{1} C_{2}-C_{3} C_{4}} \sigma
\end{aligned}
$$

As $\varphi$ goes to zero (i.e. $\varphi \rightarrow 0$ ), the liquid-bridging force $f_{a}$ also approaches to zero but not to a constant as pointed out by Ref. 22 and 14. This is in consistent with and not in conflict with the practical physical phenomenon. Nevertheless, if a approximate cylindrical profile instead of an accurate meniscus profile is used to calculate the profile area or the volume of liquid, a confliction will occur. This can be addressed as follows.

When the thickness of liquid film is very small, the curvature radius will be much smaller than the radius of sphere, i.e. $r \ll R$. In this case, instead of the practical meniscus profile, an approximate cylindrical profile can be used as in Ref. 1. The profile area described by (34) (in which $r_{1} \ll r_{2}$ ) may be approximately written as 


$$
A_{\text {lvp }}=2 \pi R \sin \varphi(D+d)
$$

where $D$ is the distance between the sphere and the plane and $d$ is the height of the sphere cap (the same as in Ref. 1).

Substituting Equations (61) and (53) into Equation (60), neglecting the surface tension (the third term of Equation (60)), considering $\theta_{b}=\theta_{t}=\theta$ and $\rho=R \sin \varphi$, and taking $D=0$ and the limit of $\varphi \rightarrow 0$, leads to

$$
f_{a}=4 \pi R \sigma \cos \theta
$$

This is the conventional Equation. Because Equation (62) is independent of the filling angle $\varphi$, this expression shows that the liquid-bridging force approaches to a constant as $\varphi$ goes to zero. Of course, this is in conflict with the fact that there is no liquid-bridging force between two completely dry surfaces.

In terms of Ref. 1, the wetted area on the plane can be approximately written as

$$
A_{l s b}=\pi R^{2} \sin ^{2} \varphi
$$

Equation (49) can be approximately rewritten as

$$
\delta x=R \cos \varphi \delta \varphi
$$

The conservation condition of volume results approximately in

$$
\delta x=\frac{A_{l s b}}{A_{\text {lvp }}} \delta z
$$

Substituting Equations (61), (63) and (64) into Equation (65), leads to

$$
\frac{\delta \varphi}{\delta z}=\frac{\tan \varphi}{2(D+d)}=\frac{\tan \varphi}{2 R(1-\cos \varphi)(1+D / d)}
$$

Deriving Equations (53), (61) and (63) with respect to the vertical coordinate $z$ and substituting Equation (66) into them, leads to

$$
\begin{gathered}
\frac{\delta A_{l s t}}{\delta z}=-\frac{2 \pi R^{2} \sin \varphi \tan \varphi}{2 R(1-\cos \varphi)(1+D / d)} \\
\frac{\delta A_{l s b}}{\delta z}=-\pi R^{2} 2 \sin \varphi \cos \varphi \frac{\tan \varphi}{2 R(1-\cos \varphi)(1+D / d)} \\
\frac{\delta A_{l v p}}{\delta z}=2 \pi R \sin \varphi
\end{gathered}
$$

Substituting Equations (67), (68) and (69) into Equation (17) and noting $\sigma_{l s t}=-\sigma \cos \theta, \sigma_{l s b}=-\sigma \cos \theta$ and $\sigma_{l v p}=\sigma$, leads to

$$
f_{a}=\pi R \sigma \cos \theta \frac{(1+\cos \varphi)^{2}}{\cos \varphi(1+D / d)}+2 \pi R \sigma \sin \varphi
$$

If the surface tension is negligible, Equation (70) can be rewritten as

$$
f_{a}=\pi R \sigma \cos \theta \frac{(1+\cos \varphi)^{2}}{\cos \varphi(1+D / d)}
$$

This is the same as that obtained by He, et al. in Ref. 1 . Although Equation (71) is not independent of the filling angle $\varphi$, the same form as Equation (62) can be obtained. This is because an approximate cylindrical profile is also used for calculation of the liquid volume. As $D=0$ (as shown in Figure 7(b)) and $\varphi \rightarrow 0$, Equation (71) changes to

$$
f_{a}=4 \pi R \sigma \cos \theta
$$

This is the same as the conventional Equation (62).

It should be pointed out that, Equation (72) is appropriate only for large sphere and thin liquid film. Although Equation (71) has been partially modified relative to Equation (72), it is appropriate only for a thin liquid film and a small deep-wide ratio, i.e. $\zeta \ll 1$.

The model built in this article is appropriate not only for the large sphere and thin liquid film, but also for the small sphere and large separating distance.

\subsection{The Edge-Effect Resulting From the Profile of the Top Solid in the Liquid-Bridge System}

In a general liquid-bridge system, the liquid will not only wet the major surface of top solid but also wet the profile near the edge of it. In this case, the pull-off force will be caused not only by the Laplace negative pressure $F_{p}=2 \sigma / r_{m} \cdot A$ and the profile surface tension of liquid $F_{\gamma}=\sigma \cdot l \cdot \sin \theta$ (e.g. as shown in Equations (16) and (24)) but also by the surface tension of liquid on the profile near the edge of top solid. The former as shown in Figure 8 can be expressed by

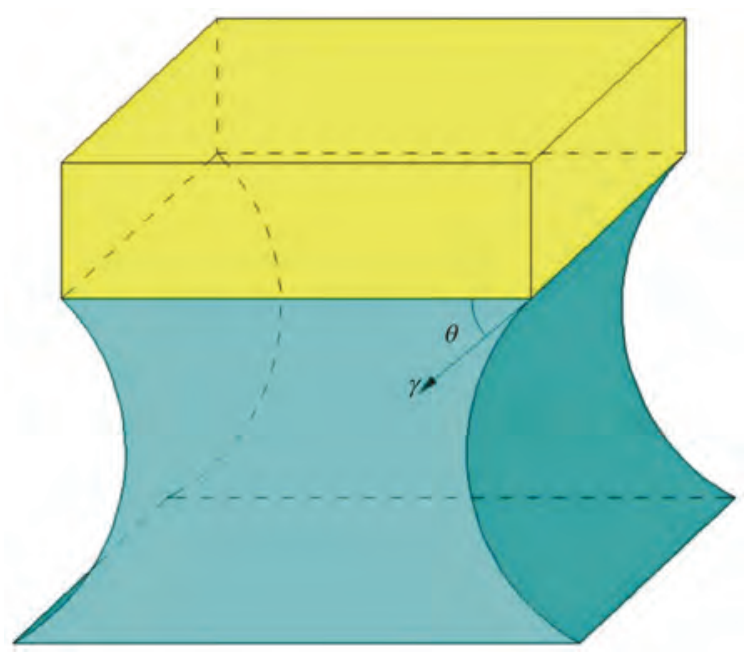

Figure 8. The major surface is wetted. 


$$
F_{T}=F_{p}+F_{\gamma}=\frac{2 \sigma}{r_{m}} \cdot A+\sigma \cdot l \cdot \sin \theta
$$

where $A$ stands for the wetted area of top solid and $\sigma$ is the surface tension of liquid $\theta$ is the contact angle of the liquid with respect to the top solid, and $l$ is the perimeter of the wetted area $A, r_{m}$ is the mean radii of the meniscus profile.

The latter as shown in Figure 9 can be expressed by

$$
F_{E}=\sigma \cdot l \cdot \cos \theta
$$

The total pull-off force as shown in Figure $\mathbf{1 0}$ can be expressed by

$$
F=\frac{2 \sigma}{r_{m}} \cdot A+\sigma \cdot l \cdot \sin \theta+\sigma \cdot l \cdot \cos \theta
$$

\section{Experiments and Comparisons}

To validate the above model in which the edge effect is considered, a series of experiments are conducted. One kind of them is to take water from a water cup as shown in Figure 11. The other kind is to pull the water from a silicon substrate plate as shown in Figure 12. The shapes of top silicon plate include rectangular and square. The areas of top silicon plates range from $200 \mathrm{~mm}^{2}$ to 400 $\mathrm{mm}^{2}$. When the amount of the water is enough, the same

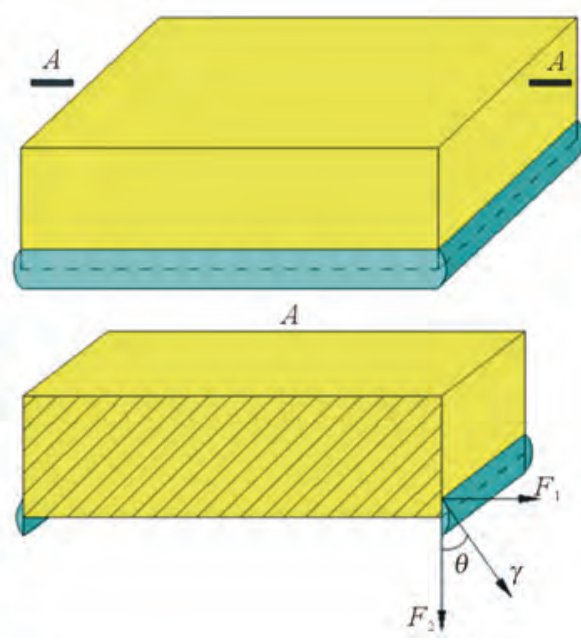

Figure 9. The edge is wetted.

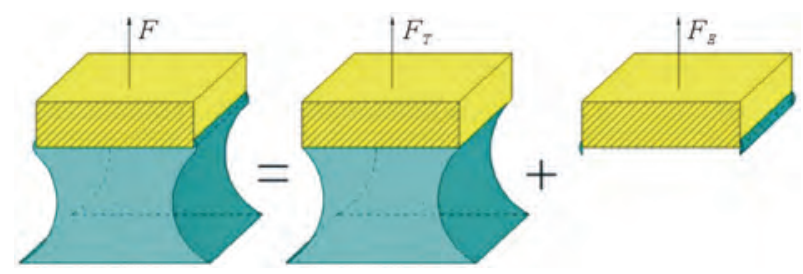

Figure 10. The total pull-off force comes from the major surface and the edge profile.

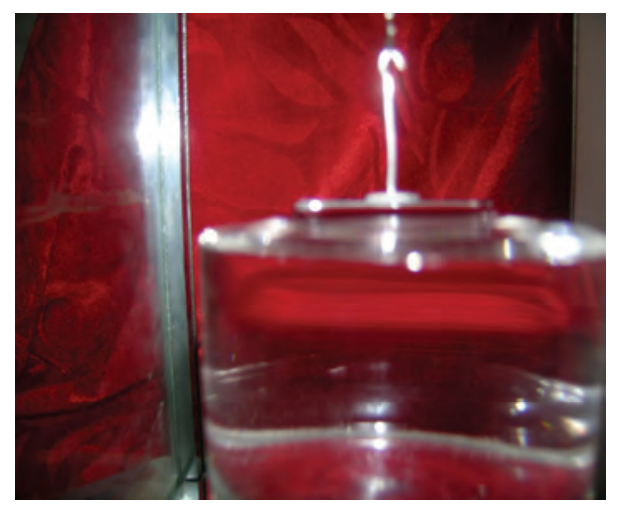

Figure 11. Taking water from cup.

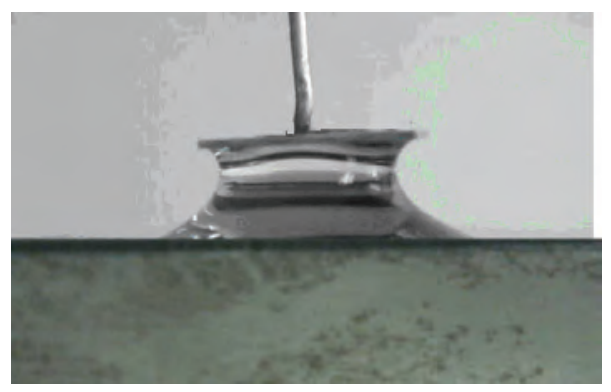

Figure 12. Taking water from the silicon substrate.

results may be obtained for both kinds of experiments. The relationship curves between the pull force and the displacement of top silicon plate are shown in Figure 13. The used top silicon plate is a rectangular plate which has $20 \mathrm{~mm}$ length and $15.71 \mathrm{~mm}$ width. Figure 13 includes seven curves corresponding the different test times. It is clear that they are very consistent and have very little deviation. The peak value of the curve is considered as pull-off force of the liquid-bridge system. It is found that the pull-off force depends strongly on the area of the top silicon plate. The corresponding curves are shown in Figure 14. In Figure 14 the calculated curves

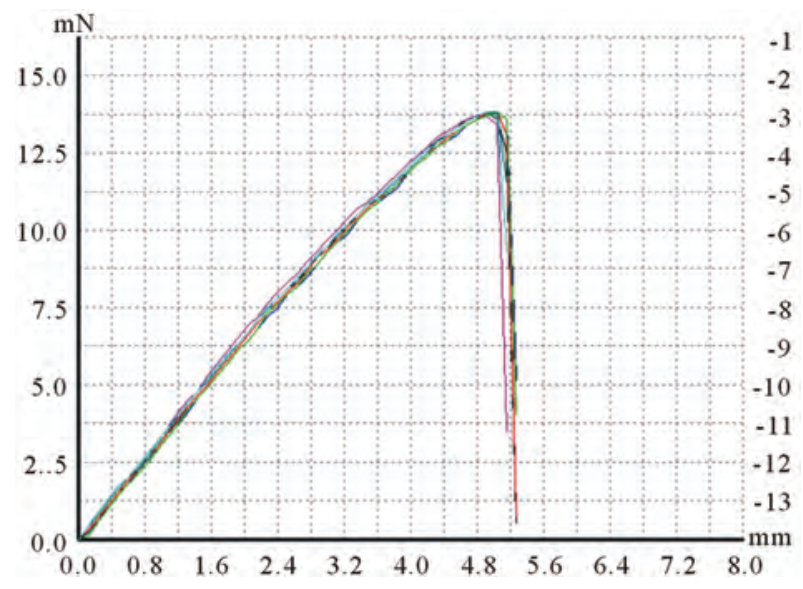

Figure 13. The curves of taking force vs displacement. 


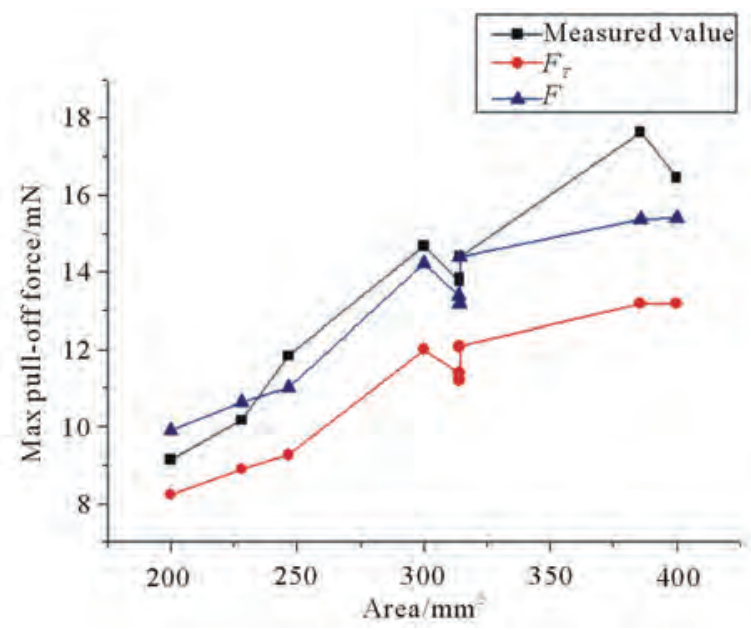

Figure 14. The curves of pull-off forces vs areas.

Table 1. Several kinds of calculated pull-off force results and the measured pull-off force.

\begin{tabular}{|c|c|c|c|c|c|c|c|}
\hline No. & $\begin{array}{l}\text { Size } \\
\text { (Length } \times \\
\text { Width) }\end{array}$ & $F_{P}$ & $F_{\gamma}$ & $F_{T}$ & $F_{E}$ & $F$ & $\begin{array}{l}\text { Measured } \\
\text { value }\end{array}$ \\
\hline 1 & $10 \times 20$ & 4.195 & 4.03 & 8.225 & 1.677 & 9.902 & 9.13 \\
\hline 2 & $11.4 \times 20$ & 4.666 & 4.218 & 8.884 & 1.755 & 10.639 & 10.16 \\
\hline 3 & $15.7 \times 15.7$ & 5.044 & 4.218 & 9.262 & 1.755 & 11.017 & 11.84 \\
\hline 4 & $30 \times 10$ & 6.624 & 5.373 & 11.997 & 2.235 & 14.232 & 14.67 \\
\hline 5 & $15.71 \times 20$ & 6.591 & 4.797 & 11.388 & 1.996 & 13.384 & 13.76 \\
\hline 6 & $31.42 \times 10$ & 6.591 & 5.564 & 12.155 & 2.315 & 14.47 & 14.4 \\
\hline 7 & $\begin{array}{l}17.73 \times \\
17.73\end{array}$ & 6.443 & 4.763 & 11.196 & 1.982 & 13.178 & 13.86 \\
\hline 8 & $\begin{array}{l}19.64 \times \\
19.64\end{array}$ & 7.894 & 5.277 & 13.171 & 2.195 & 15.366 & 17.62 \\
\hline 9 & $20 \times 20$ & 7.805 & 5.373 & 13.178 & 2.235 & 15.413 & 16.45 \\
\hline
\end{tabular}

by different models are also given. It can be seen that, the results from the model which considering the edge effect are more close to the testing results. In addition, more information including $F_{p}$ and $F_{\gamma}$ are given in Table 1.

\section{References}

[1] M. He, A. S. Blum, D. E. Aston, C. Buenviaje and R. M. Overney, "Critical Phenomena of Water Bridges in Nanoasperity Contacts,” Journal of Chemical Physics, Vol. 114, No. 3, 2001, pp. 1355-1360. doi:10.1063/1.1331298

[2] J. Jang, G. C. Schatz and M. A. Ratner, "Capillary Force in Atomic Force Microscopy," Journal of Chemical Physics, Vol. 120, No. 3, 2004, pp. 1157-1160. doi:10.1063/1.1640332

[3] T. Stifter, O. Marti and B. Bhushan, “Theoretical Inves- tigation of the Distance Dependence of Capillary and Van der Waals Forces in Scanning Force Microscopy," Physical Review B, Vol. 62, No. 20, 2000, pp. 13667-13673. doi:10.1103/PhysRevB.62.13667

[4] H. Fan and Y. X. Gao, "Elastic Solution for Liquid-bridginginduced Microscale Contact," Journal of Applied Physics, Vol. 90, No. 12, 2001, pp. 5904-5910. doi:10.1063/1.1415057

[5] A. Chau, S. Regnier, A. Delchambre and P. Lambert, "Influnce of Geometrical Parameters on Capillary Forces," Proceedings of the 2007 IEEE International Symposium on Assembly and Manufacturing, Ann Arbor, 22-25 July 2007, pp. 215-220.

[6] D. Wu, N. Fang, C. Sun and X. Zhang, "Stiction Problems in Releasing of 3D Microstructures and Its Solution," Sensors and Actuators A: Physical, Vol. 128, No. 1, 2006, pp. 109-115. doi:10.1016/j.sna.2005.12.041

[7] P. Lambert, F. Seigneur, S. Koelemeijer and J. Jacot, “A Case Study of Surface Tension Gripping: The Watch Bearing," Journal of Micromechanics and Microengineering, Vol. 16, No. 7, 2006, pp. 1267-1276. doi:10.1088/0960-1317/16/7/021

[8] J. S. McFarlane and D. Tabor, "Adhesion of Solids and the Effect of Surface Films," Proceedings of the Royal Society A, London, July 1950, pp. 224-243. doi:10.1098/rspa.1950.0096

[9] F. P. Bowden and D. Tabor, "Friction and Lubrication of Solids, Part I,” Oxford University Press, London, 1954.

[10] F. P. Bowden and D. Tabor, "Friction and Lubrication of Solids, Part II,” Oxford University Press, London, 1964.

[11] B. Bhushan, "Principles and Applications of Tribology," Wiley, New York, 1999.

[12] F. M. Orr, L. E. Scriven and A. P. Rivas, "Pendular Rings between Solids: Meniscus Properties and Capillary Force,” Journal of Fluid Mechanics, Vol. 67, No. 4, 1975, pp. 723-742. doi:10.1017/S0022112075000572

[13] B. Zhang and A. Nakajima, "Nanometer Deformation Caused by the Laplace Pressure and the Possibility of Its Effect on Surface Tension Measurements," Journal of Colloid and Interface Science, Vol. 211, No. 1, 1999, pp. 114-121. doi:10.1006/jcis.1998.5978

[14] O. H. Pakarinen, A. S. Foster, M. Paajanen, T. Kalinainen, J. Katainen, I. Makkonen, J. Lahtinen and R. M. Nieminen, "Towards an Accurate Description of the Capillary Force in Nanoparticle-surface Interactions,” Modelling and Simulation in Materials Science and Engineering, Vol. 13, No. 7, 2005, pp. 1175-1186. doi:10.1088/0965-0393/13/7/012

[15] H. Shinto, K. Uranishi, M. Miyahara and K. Higashitani, "Wetting-induced Interaction between Rigid Nanoparticle and Plate: A Monte Carlo Study," Journal of Chemical Physics, Vol. 116, No. 21, 2002, pp. 9500-9509. doi:10.1063/1.1473817

[16] C. Gao, “Theory of Menisci and Its Applications,” Applied Physics Letters, Vol. 71, No. 13, 1997, pp. 1801-1803. doi:10.1063/1.119403

[17] L. R. Fisher and J. N. Israelachvili, "Direct Measurement 
of the Effect of Meniscus Forces on Adhesion: A Study of the Applicability of Macroscopic Thermodynamics to Microscopic Liquid Interfaces,” Colloids and Surfaces, Vol. 3, No. 4, 1981, pp. 303-319.

doi:10.1016/0166-6622(81)80058-3

[18] H. K. Christenson, "Adhesion between Surfaces in Unsaturated Vapors - A Reexamination of the Influence of Meniscus Curvature and Surface Forces," Journal of Colloid and Interface Science, Vol. 121, No. 1, 1988, pp. 170-178. doi:10.1016/0021-9797(88)90420-1

[19] H. K. Christenson and V. V. Yaminsky, “Adhesion and Salvation Forces between Surfaces in Liquids Studied by Vapor-Phase Experiments,” Langmuir, Vol. 9, No. 9, 1993, pp. 2448-2454. doi:10.1021/la00033a030

[20] J. P. Kirkness, H. K. Christenson, J. R. Wheatley and T. C. Amis, "Application of the 'Pull-Off' Force Method for
Measurement of Surface Tension of Upper Airway Mucosal Lining Liquid," Physiological Measurement, Vol. 26, No. 5, 2005, pp. 677-688. doi:10.1088/0967-3334/26/5/009

[21] J. Yuan, Z. Shao and C. Gao, "Alternative Method of Imaging Surface Topologies of Nonconducting Bulk Specimens by Scanning Tunneling Microscopy,” Physical Review Letters, Vol. 67, No. 7, 1991, pp. 863-866. doi:10.1103/PhysRevLett.67.863

[22] H. Fan and G. F. Wang, "Stability Analysis for Liquidbridging Induced Contact," Journal of Applied Physics, Vol. 93, No. 5, 2003, pp. 2554-2558. doi:10.1063/1.1544652

[23] S. Gao and H. Liu, “Capillary Mechanics,” Science Press, Beijing, 2010. 